\title{
Focus on Affinity Mass Spectrometry
}

$\mathrm{A}$ lthough the application of affinity techniques in the analysis of biopolymers by mass spectrometry has become an established approach over the last decade or so, it is re-emerging as new methodology combinations and tools for the structure identification and quantification of biopolymer interactions become available. It can be productively used for the identification of proteins and protein complexes from biological material and for the enrichment and analysis of protein structure modifications. These approaches may be viewed as examples of "affinity mass spectrometry"; they are presently developing as valuable tools for a variety of important research areas, from identifying the recognition epitopes involved in biomolecular interactions and quantitatively determining interactions, to utilizing them in personalized medicine.

This focus gives an assessment of the current state of affinity mass spectrometry by presenting a compilation of articles that illustrate both methods and applications. Included are descriptions of approaches for studying antibody-peptide epitopes and quantifying their affinities, for identifying post-translational modifications, for mapping epitopes by chemical reactions, for improving the specificity of analytical proteomics, and for solving problems in personalized medicine. The "marriage" of affinity-detection techniques with the structure determination capability of MS continues to open the door to powerful new applications in biochemistry, immunology and biomedicine. These developments indicate that the "marriage of affinity and MS" will provide new information than cannot be provided by other methods, and not by any single methodology.

We selected seven articles ranging from method development to application to comprise this focus. In the first article, Przybylski and coworkers report a new, on-line bioaffinity ESI mass spectrometry instrumental combination for examining protein/peptide and other biopolymer interactions. The biosensor is a surface acoustic wave device that may compete with surface plasmon resonance, which up to now is most widely used in investigating biomolecular interactions. The approach allows affinity constants to be determined and enables bioanalytical applications to the simultaneous, label-free determination and quantification of biopolymer-ligand interactions as diverse as antigenantibody and lectin-carbohydrate complexes.

Continuing the theme of method development, Deterding and coworkers report a useful evaluation of several affinity resins consisting of ionic metals or metal oxides for their capabilities to enrich for phosphopeptides prior to MS analysis. The authors compared commercially available enrichment metal oxide or metal media to provide a guide to select the most efficient and specific method that can be implemented as a standard enrichment procedure in phosphopeptide analysis.

The next contribution reports a joint effort by the Gross and Przybylski laboratories to adapt the PLIMSTEX method (protein-ligand interactions by MS, titration, and H/D exchange) to examine interactions of antibodies with tyrosine- nitrated peptides, an important posttranslational modification in oxidative stress. One motivation is to conserve valuable protein material, which is especially important when investigating large proteins. Their approach is a dilution strategy whereby the extent of H/D amide exchange of the peptide is measured as a function of diluting the original antibody/ antigen solution. The results are validated in comparison with data from the standard PLIMSTEX approach and from the literature.

In the final method development contribution, the problem of lack of robustness and reproducibility in quantitative mass spectrometry-based proteomic assays is addressed by Savitski, Bantscheff, and coworkers. They describe a targeted mass spectrometric data acquisition strategy for affinity enriched sub-proteomes. The approach enables a substantially improved reproducibility of detection and improved quantification via isobaric tags (e.g., iTRAQ). Multiple fragmentation of the same peptides resulted in better statistics and more precise reporter-ion-based quantification. With the targeted data acquisition approach, they were able to quantify more proteins and obtain higher precision than previously.

The next contribution is a compelling example showing the transition of method development to application. Borchers and coworkers describe an immuno MALDI MS-based assay of the renin-angiotensin-aldosterone system that plays an important role in blood-pressure regulation. Immuno-MALDI allows angiotensin I and a stable, isotopically labeled standard peptide to be captured by taking advantage of affinity on immobilized anti-peptide antibodies. The sample is then analyzed by MS. The immuno MALDI assay of angiotensin I may become a routine, rapid, and specific approach for determining plasma rennin activity and assist in the management of high blood pressure.

Completing the theme of applications, we present a contribution from the Tomer group wherein they investigate a subset of the neutralizing anti-HIV antibodies that recognize epitopes on the envelope protein of the human immunodeficiency virus. These epitopes are 
exposed during conformational changes that occur with binding. The researchers, using chemical mapping of lysine and arginine residues and analysis by mass spectrometry, can locate the binding epitope. More importantly, they are able to establish that the epitope is discontinuous.

The last contribution in the focus points clearly to applications in personalized medicine. Glocker and collaborators use multifactorial analysis of affinity MS data from proteins found in serum to distinguish pregnant women with preeclampsia from control individuals. They fractionated serum proteins using affinities to reversed-phase material coated on magnetic beads, or by fractionated precipitation. Analysis of six protein signals by MS showed that sorting of the individual spectra can be achieved with high accuracy and that patients with preeclampsia can be distinguished rapidly from those who do not have the disease.

We hope that the present assembly of articles brings useful insight into affinity mass spectrometry. This new combination (or "hyphenated") area will be illustrated further by a Critical Insight of the same theme in a subsequent issue of the journal. It is an area where affinity measurements and approaches not only are important in their own right, but also provide a basis for chemical analysis, combining the specificity of affinity determination with the specificity, speed, and sensitivity of mass spectrometry.

Michael L. Gross Washington University in St. Louis

Michael Przybylski University of Konstanz 